\title{
Automotive Radar and Lidar Systems for Next Generation Driver Assistance Functions
}

\author{
R. H. Rasshofer and K. Gresser \\ BMW Group Research and Technology, Hanauer Str. 46, 80992 Munich, Germany
}

\begin{abstract}
Automotive radar and lidar sensors represent key components for next generation driver assistance functions (Jones, 2001). Today, their use is limited to comfort applications in premium segment vehicles although an evolution process towards more safety-oriented functions is taking place. Radar sensors available on the market today suffer from low angular resolution and poor target detection in medium ranges ( 30 to $60 \mathrm{~m}$ ) over azimuth angles larger than $\pm 30^{\circ}$. In contrast, Lidar sensors show large sensitivity towards environmental influences (e.g. snow, fog, dirt). Both sensor technologies today have a rather high cost level, forbidding their wide-spread usage on mass markets.

A common approach to overcome individual sensor drawbacks is the employment of data fusion techniques (BarShalom, 2001). Raw data fusion requires a common, standardized data interface to easily integrate a variety of asynchronous sensor data into a fusion network. Moreover, next generation sensors should be able to dynamically adopt to new situations and should have the ability to work in cooperative sensor environments.

As vehicular function development today is being shifted more and more towards virtual prototyping, mathematical sensor models should be available. These models should take into account the sensor's functional principle as well as all typical measurement errors generated by the sensor.
\end{abstract}

\section{Present status}

\subsection{Introduction}

Radar and Lidar systems play an important role in modern premium segment vehicular technology (Schneider, 1999; Jones, 2001). Today, vehicle applications mainly represent comfort functions (e.g. ACC) although an evolution from comfort towards safety functions has started recently. Functional requirements for vehicular safety systems impose much more stringent requirements on next generation sensor systems than typically found in today's sensors. With stateof-the-art sensor technology available on the market today, a

Correspondence to: R. H. Rasshofer

(ralph.rasshofer@bmw.de) number of safety functions can only be realized with limited performance.

Today (2004), the over-all market penetration of long range vehicular perception sensors is very low and mainly restricted to vehicles of the premium segment. Most sensors are employed to realize simple ACC (adaptive cruise control) functions based on $77 \mathrm{GHz}$ radar technology although some lidar-based ACC systems have shown up on the Japanese market recently. In ACC systems, commonly long range sensors with a detection range larger than $150 \mathrm{~m}$ on cars and an azimuth coverage of \pm 6 to $\pm 8^{\circ}$ are employed. These sensors are optimised for very smooth distance regulation and thus have rather poor dynamic performance. Typical latency values for new target detection are 250 to $500 \mathrm{~ms}$, internal filtering commonly limits target dynamics to values below $\pm 4 \mathrm{~m} / \mathrm{s}^{2}$. Given these facts, ACC sensors available on the market cannot be used for dynamic cruise control applications like e.g. city traffic or stop-and-go without major adjustments in sensor firmware and hardware (Widmann, 2000).

\subsection{New vehicle functions}

Car manufacturers world wide have successfully implemented a number of new vehicle functions based on radar and lidar sensors recently. Most of these functions will be put to mass production in the next two years. Figure 1 shows an overview of these functions and a brief description of the detection ranges typically required along with the sensor technology likely to be used. As can be seen, today there is no clear trend towards using a specific sensor technology, radar and lidar systems are equally employed in a variety of applications. Sometimes a combination of multiple sensors is used to achieve the detection range required by the vehicle function. Usage of sensors available for mass production 2 to 3 years before the production start of the vehicle is typical for all applications listed here.

\subsection{Limits of today's radar sensors}

\subsubsection{Azimuth measurement}

Radar sensors available for production today typically have high measurement accuracy in range. The range resolution 


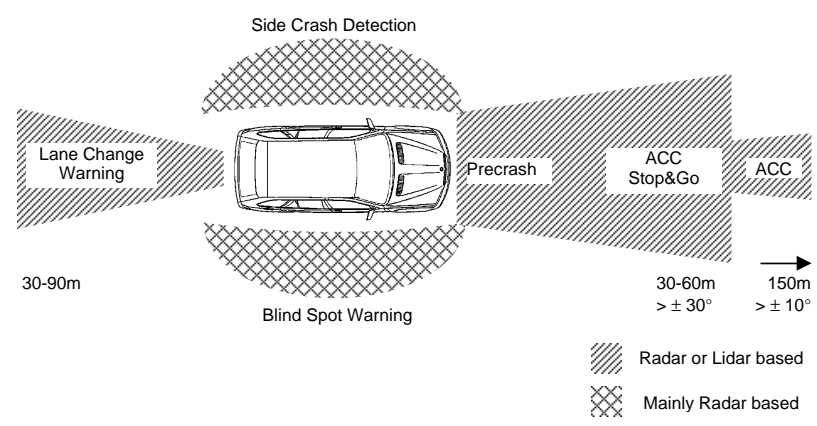

Fig. 1. New radar- and lidar-based vehicle functions likely to be available on the market in the next two years.

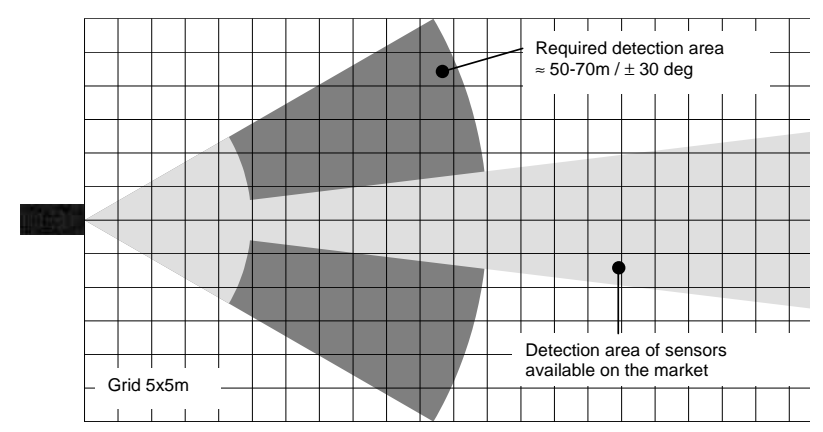

Fig. 2. Sensor coverage for front-looking applications like e.g. ACC stop \& go: Requirements vs. radar sensors available.

practically achieved is a factor of 1.3 to 2.0 larger than the physical limit implied by the signal bandwidth and usually is good enough for most applications. The main drawback of today's radar sensors is found in the measurement principles used for azimuth angle determination. As radar sensors employing mechanically scanning antennas - which have been used wide spread in the early days of automotive radar - have disappeared from the market almost completely, only electronic azimuth measurement principles like "sequential lobing" (Flacke, 1999) or "monopulse" (Li, 1999) are found in today's sensors. Both principles offer the possibility to measure a target's azimuth angle relatively to the sensor, but only work unambiguously in case of a single target. Generally speaking, both principles have a lack of angular resolution, i.e. cannot resolve two targets in the same range having different azimuth. Sensor manufacturers overcame this problem by means of tracking and filtering. As tracking and filtering methods rely on target model assumptions, critical detection errors tend to take place in certain situations, leading to target loss, wrong measurement of target's azimuth or even detection of ghost targets. These errors tend to appear rather seldom but with a finite probability so safety-critical decisions cannot only rely on information provided by today's radar sensors alone.

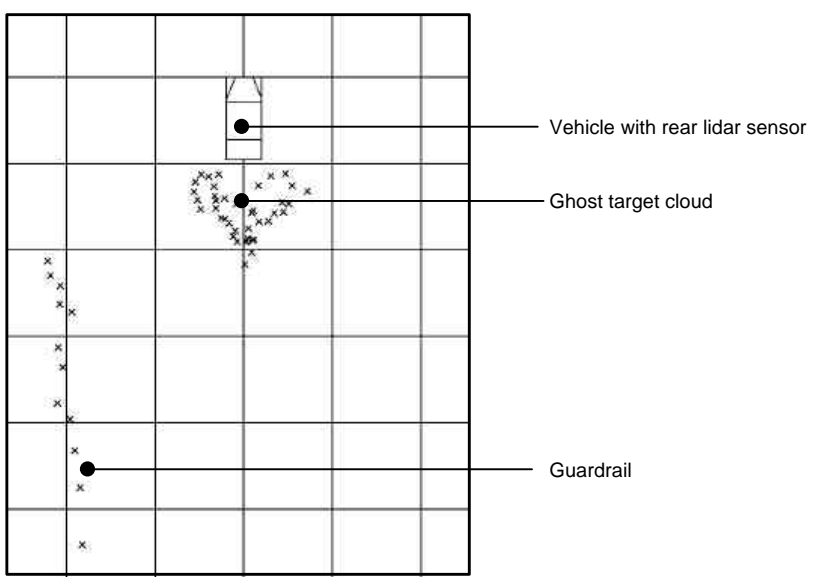

Fig. 3. Lidar ghost target cloud generated by water spray on a wet autobahn during heavy rain. Each point represents a target detected by the lidar. Grid: $5 \times 5 \mathrm{~m}$.

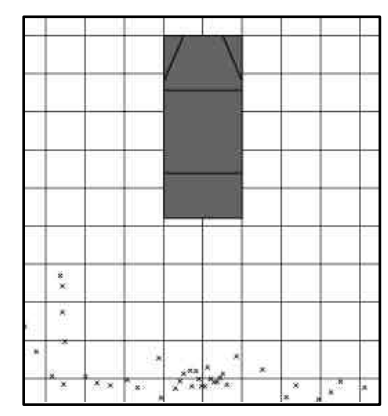

Grid: $1 \times 1 \mathrm{~m}$

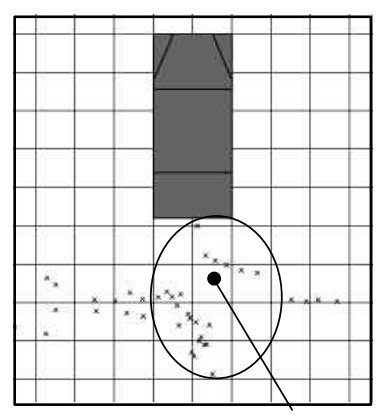

Excessive Measurement Errors
Fig. 4. Ghost target generation in a lidar system exceeding its dynamic range. Left: $4 \mathrm{~m}$ distance to highly reflective wall, no measurement errors. Right: $2 \mathrm{~m}$ distance to highly reflective wall, measurement errors increasing.

\subsubsection{Detection area}

Concerning front-looking applications like e.g. ACC stop \& go, the detection of cutting-in vehicles represents a special challenge. To clearly classify the cutting-in case, target vehicles have to be detected in a distance of 50 to $70 \mathrm{~m}$ from the sensor on both adjacent lanes. This transfers to a desired detection azimuth of $\pm 30^{\circ}$ over 50 to $70 \mathrm{~m}$ distance which cannot be realized with any sensor on the market today. Figure 2 illustrates this situation. Next generation sensors should be enhanced in sensitivity to cover the important medium range area.

\subsubsection{Frequency allocation}

Well-known physical laws (1) relate radar signal bandwidth $B$ to a radar's range resolution $\delta \mathrm{R}$ :

$\delta R>c_{0} / 2 B$ 
Typically, short range radars require high range resolution so ultra wide band sensors are commonly found in these applications (Rollmann, 2004, Gresham 2004). If cost-effective $24 \mathrm{GHz}$ ISM band operation is desired, legal restrictions varying from country to country have to be taken into account. On a world-wide basis, only a few $\mathrm{MHz}$ of bandwidth are free to use. In the USA, FCC has allowed the use of ultra wideband radar sensors since 2002 (FCC, 2002) however in the EC, use of ultra wideband sensors is likely to be limited to a maximum of $7 \%$ fleet penetration and stop of new sensor deployment by end of 2012 .

A long term solution to the limited bandwidth problem of $24 \mathrm{GHz}$ near distance sensors will be the use of the recently allocated $79 \mathrm{GHz}$ millimetre wave band spreading from 77 to $81 \mathrm{GHz}$. Sensors at these frequencies will be available not before 2015 in mass production since neither low-cost millimetre wave technology nor mass production facilities will be ready before that date.

\subsection{Limits of today's lidar sensors}

\subsubsection{Lidar technology today}

Today, lidar sensors can be divided into two groups: multibeam lidars and scanning lidars. While multibeam lidars employ an array of transmitting and receiving elements illuminating separate angular sections, scanning lidars usually use one transmitter and receiver with a mechanically scanned lens system (e.g. rotating mirror or prism). The azimuth of the rotating scan system directly represents the target azimuth in case of detection.

All lidars available on the market today operate in the near infrared. Their transmit output power level is limited by eye safety constrains. As the receiver sensitivity of a lidar sensor is limited by the detection diode (PIN diode or avalanche diode) operating close to the physical limits today, maximum sensor range is mainly influenced by lens aperture and hence by the size of the sensor.

\subsubsection{Environmental conditions}

Lidar sensors employ laser light for distance measurement. Atmospheric damping influences the maximum detection range, rain, snow and fog typically limit the lidar's detection range to the same performance a human eye has under these conditions. Given automotive environment, water spray of a preceding vehicle for front-looking applications or spray from the own vehicle for back-looking applications further limits the sensor performance by introducing ghost targets (see Fig. 3). Moreover, dirt and ice coverage on the sensor will stop its proper operation, however these effects can easily be detected by the sensors itself and thus can be signalled to the application.

\subsubsection{Dynamic range}

As object corners represent sharp boundaries for laser light, very large signal dynamic between adjacent lidar beams is

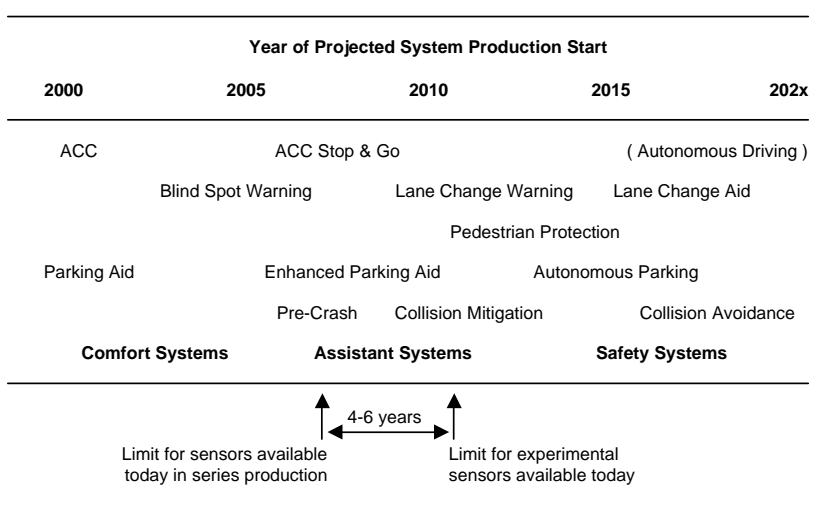

Fig. 5. General roadmap for future vehicle functions (not OEM specific).
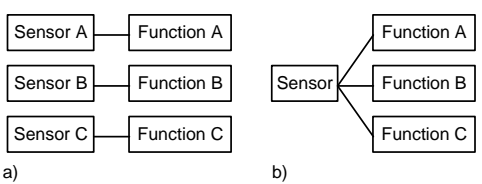

b)

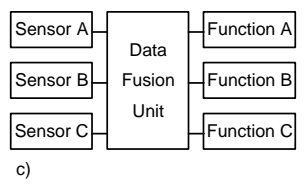

Fig. 6. Sensor system architectures. (a) Separate systems (present situation) (b) Multiple use of sensors, (c) Multi sensor data fusion.

noticed. To cope with this, dynamic intra-pulse gain control has to take place. If the maximum dynamic range of a lidar is exceeded, typically heavy generation of ghost targets along with real target losses starts due to non-linearity in the photo detector diode and compression of the video amplifier chain. Figure 4 shows an example of this phenomena.

\section{Sensor requirements for next generation vehicular systems}

\subsection{General roadmap for future vehicle functions}

Figure 5 shows a general roadmap for future vehicle functions valid for many car manufacturers. The roadmap displays the global trend of evaluation from comfort system via driver assistance functions towards pronounced safety features. The realization of these different functions today is mainly limited by the quality of sensors available. Due to this, the functions can be divided into three different groups:

1. Functions that can be realized with mass-production ready sensors available today

(e.g. parking aid or ACC)

2. Functions which can be realized as demonstrators using experimental high-end sensors (e.g. lane change warning or collision mitigation systems)

3. Functions that cannot be realized at all due to quality lacks of the corresponding sensor systems (e.g. collision avoidance). 
Table 1. Typical strengths and weaknesses of automotive sensors available today.

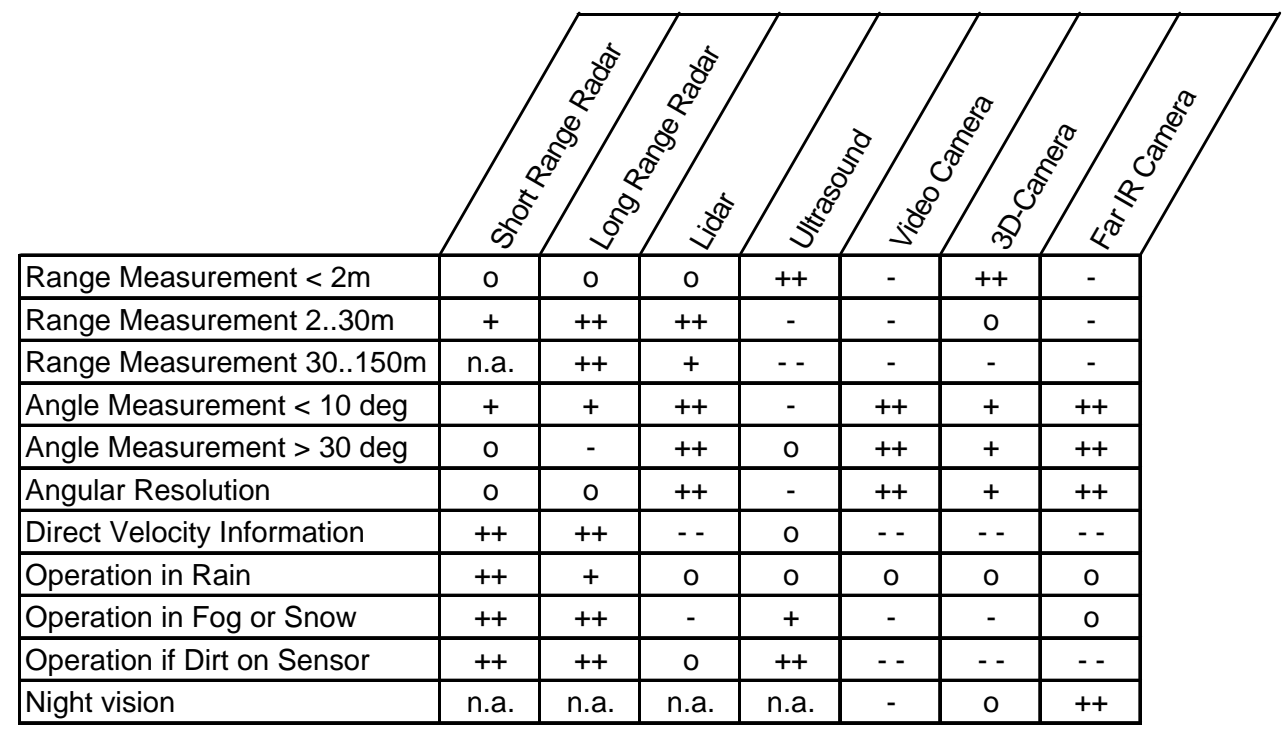

++ : Ideally suited / + : Good performance / o : Possible, but drawbacks to be expected;

- : Only possible with large additional effort / - - : Impossible / n.a. : Not applicable

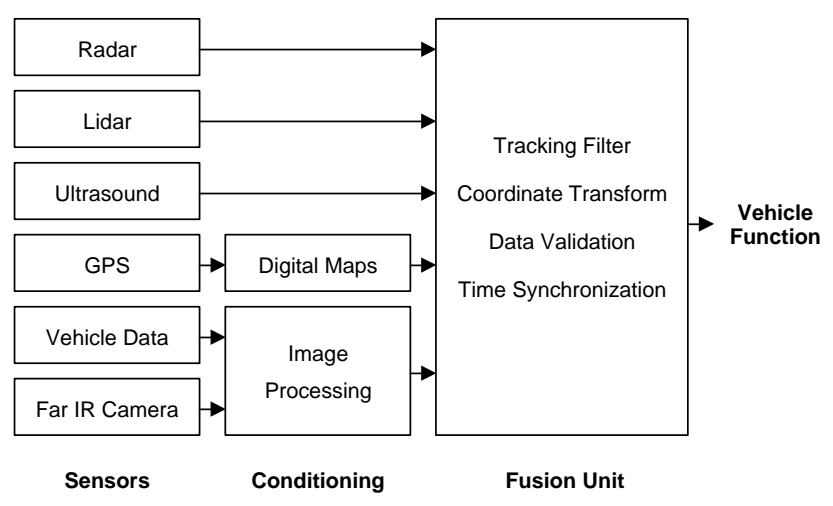

Fig. 7. Typical automotive sensor data fusion architecture.

Typically, proof of function has to take place 4 to 6 years before the start of vehicle production. By then, the step from an experimental sensor system towards a mass-production ready, automotive qualified part has to take place at the tier 1 supplier.

\subsection{Trends in vehicle function architecture}

Today, each vehicle application uses its own, functionally optimised sensor (Fig. 6a). In the future, this situation might change since the variety of new vehicle functions will not allow for separate sensor systems due to cost and space restrictions. One possible scenario will be the use of one sensor system by more than one vehicle function (e.g. re-use of ACC stop \& go sensors for pre-crash application and pedestrian protection, see Fig. 6b). This scenario requires the same sensors to be operated in different applications sometimes having diverting requirements which usually imposes an enhanced quality level on the sensor.

As vehicle function development today mainly is limited by sensor quality, means to enhance sensor system performance have been discussed extensively. Related to their operation principle, different sensor technologies tend to show complementary strengths in measuring certain object parameters. Table 1 gives an overview of typical strengths and weaknesses for automotive sensors available today. As can be seen, strengths of different sensor types (e.g. radar and camera systems ) can be combined to end up with an improved sensor system: This represents the concept of multi sensor data fusion shown in Fig. 6c. To practically perform data fusion, a multi step process has to take place. Figure 7 shows an overview of a typical multi sensor data fusion architecture. As far as the interface of the sensors to the fusion unit is concerned, certain restrictions apply. The sensors should provide a standardized object data interface which comprises only minimal and transparent data pre-processing and tracking. Moreover, the sensors should provide a precise measurement timestamp which enables the fusion unit to process asynchronous measurement data. Most sensors have the ability to internally judge a measured value with respect to the quality of measurement. This is obvious for radar and lidar where e.g. a good signal-to-noise ratio (SNR) usually indicates trustable measurements while a bad SNR makes measured values questionable. If these quality measures are available, they must be transmitted to the fusion unit to make probabilistic weighting of different measurements possible (Houles, 1989). 


\subsection{Sensor modelling}

As vehicle functions emerge into more safety critical applications, function development and verification becomes increasingly more difficult. Testing the system to its limits during qualification and functional optimisation often means to provoke sensor or system failure. This might result in dangerous situations for the test engineers. If parameters should be tuned for the average driver, many non-skilled tests persons have to check the function during test sessions using experimental prototype systems. These tests often are impossible to be done in reality since they would endanger test persons or would require set up of artificial situations difficult to construct on test tracks. The solution to this problem is virtual function prototyping which is typically done in modern driving simulators. Although these simulators are highly developed with respect to vehicle dynamics and graphic simulation, functional development requires an accurate modelling of perception sensors which is not implemented in most simulators available up to now. Sensor modelling must include the sensors' measurement variance in range and angle, modelling of detection properties and description of sporadic sensor errors like generation of ghost targets and sporadic target losses. In the future, OEMs will require sensor manufacturers to provide virtual function models for their sensors well before first hardware samples become available. This will enable the OEM to speed up the prototyping process and to simplify the validation process.

Moreover, sensor error models provide very important input for sensor data fusion algorithms as they provide a-priori knowledge of the sensor's measurement noise covariance matrix R (Bar-Shalom 1989).

2.4 Vision: Cooperative sensors for automotive applications

On the way towards vehicle safety systems based on perception sensors, absolute reliability of sensor information becomes more and more crucial. For today's self-contained sensor systems, however, there is a limit of reliability given by the ambiguity of road scenarios combined with principle-inherent finite sensor measurement quality. A classic way to improve sensor information quality in radar and lidar is the use of cooperative targets which interact with an interrogating sensor. This concept has been successfully used in aviation (e.g. distance measurement equipment, DME) for decades and proved its feasibility to secure safety-critical decisions. Cooperative sensors could use e.g. communication properties of radar (Lindenmeier, 2003) to exchange object information between vehicles. This information could be used to check data plausibility and thus improve quality of self-contained measurements each vehicle did on its own. Moreover, communication to infrastructure (e.g. traffic lights) or beaconing of dangerous or vulnerable objects (e.g. pedestrians) could become possible.

\section{Conclusions}

Today, lidar and radar systems are mainly used in ACC comfort applications although a variety of new radar- and lidar-based vehicle functions will be introduced by OEMs in the next two years. Main drawback of today's radar sensors is their lack of angular resolution while today's lidar sensors show high sensitivity towards environmental influences. For next generation vehicle functions, quality of perception sensors is the main limiting factor. Ideally speaking, upcoming radar and lidar sensors should have improved angular resolution, should be free to use in any country and should be easily integrated into data fusion systems. Moreover, these sensors should have virtual function models available and should be able to interact with each other and with infrastructure to work in cooperative sensor environments.

\section{References}

Bar-Shalom, Y., Chang, K. C., and Blom, H. A. P.: Automatic Track Formation in Clutter with a Recursive Algorithm, Proceedings of the 28th Conference on Decision Control, Tampa, Florida, 14021408, December 1989.

Bar-Shalom, Y., Li, X., and Kirubarajan, T.: Estimation with Applications to Tracking an Navigation, John Wiley \& Sons, Inc., New York, 2001.

FCC: First report and order, revision of part 15 of the comission's rules regarding ultra wideband transmission systems, FCC, Washington, D.C., ET Docket 98-153, 2002.

Flacke, J., Heckl, K., and Kaiser, B. : Verfahren zur Winkelbemessung mittels eines Mehrfachantennenradarsystems, European Patent EP 0989416 A2, June 1999.

Gresham, I., Jenkins, A., Egri, R., et.al.: : Ultra-Wideband Radar Sensors for Short-Range Vehicular Applications, IEEE Transactions on Microwave Theory and Techniques, Vol. 52, No. 9, 2105-2122, 2004.

Houles A. and Bar-Shalom, Y.: Multisensor Tracking of a Maneuvering Target in Clutter, IEEE Transactions on Aerospace and Electronic Systems, 176-189, 1989.

Jones, W. D.: Keeping Cars from Crashing, IEEE Spectrum, September 2001.

Li, D. D., Luo, S. C., Pero, C., Wu, X., and Knox, R. M.: Millimeter-Wave FMCW/Monopulse Radar Front-End for Automotive Applications, MTT-S Int. Microwave Symp.Dig., 277280, 1999.

Lindenmeier, S., Boehm, K., and Luy, F.: A Wireless Data Link for Mobile Applications, IEEE Microwave and Wireless Components Letters, Vol. 13, No. 8, 326-328, 2003.

Rollmann, G., Knoll, P., Mekhaiel, M., Schmid, V., and Blöcher, H.-L.: Short Range Radar (SRR) - System for Automotive Application, IEEE MTT/AP German Newsletter, Vol. 8, No. 1, June 2004.

Schneider, R. and Wenger, J.: System aspects of future automotive radar, MTT-S Int. Microwave Symp. Dig., 293-296, 1999.

Widmann, G. R., Daniels, M. K., Hamilton, L., et.al.: Comparison of Lidar-Based and Radar-Based Adaptive Cruise Control Systems, SAE Technical Paper Series, 2000-01-0345, 2000. 